\title{
Adaptación y validación al español de la Escala de Empoderamiento Psicológico
}

\section{Spanish adaptation and validation of the Psychological Empowerment Scale}

\author{
Francisco D. Bretones ${ }^{1}$ y María J. Jáimez ${ }^{2}$
}

${ }^{1}$ Doctor en Psicología por la Universidad de Granada. Profesor del Departamento de Psicología Social de la Universidad de Granada. Decano de la Facultad de Relaciones Laborales y Recursos Humanos de la Universidad de Granada. E-mail: fdiazb@ugr.es

${ }^{2}$ Doctora en Ciencias Sociales por la Universidad de Granada. Directora del Área de Posgrados en Gestión de RRHH de la Escuela Internacional de Posgrados Mainjobs. Investigadora del Grupo de investigación en Salud y Bienestar Organizacional de la Universidad de Granada. E-mail:mjaimez@grupomainjobs.com

\begin{abstract}
Grupo de Investigación en Salud y Bienestar Organizacional, Facultad de Relaciones Laborales y Recursos Humanos, Universidad de Granada. Granada, España.
\end{abstract}

\section{Resumen}

El empoderamiento psicológico es un constructo de gran importancia en Psicología. Aunque existen diversos instrumentos de medida, el Psychological Empowerment Instrument de Spreitzer (PEI, 1995) ha sido tradicionalmente el más utilizado en el área de la Psicología Organizacional. Originalmente, este instrumento constaba de 16 ítems organizados en cuatro factores. Por ello, el objetivo del presente artículo fue la adaptación y validación al español del PEI, así como el análisis de su estructura factorial. Para ello, se llevaron a cabo dos estudios independientes. El objetivo del primero fue su traducción al español y su adaptación cultural en una muestra de 242 estudiantes universitarios para analizar la estructura factorial de los ítems y su fiabilidad. En el segundo estudio, se aplicó el nuevo instrumento traducido a una muestra de 317 trabajadores de una empresa de servicios con el fin de validarlo y comprobar la bondad del modelo, así como la validez concurrente con otras variables, tales como el empoderamiento estructural, el compromiso afectivo y la rotación, de la que se conoce su relación por estudios previos. El nuevo cuestionario resultante confirmó la estructura factorial original en cuatro dimensiones (competencia, significado, autonomía e impacto), aunque redujo el número de ítems (de los 16 originales a 13). El cuestionario adaptado al español obtenido tuvo, además, buenos parámetros psicométricos y de bondad de ajuste. El trabajo finaliza discutiendo sobre la relevancia del instrumento adaptado y sus posibles aplicaciones.

Palabras clave: compromiso, empoderamiento, español, intención de irse, validación de cuestionario.

\section{Abstract}

Psychological empowerment is a construct with a multifaceted character composed of four basic cognitions (impact, competence, meaning, and self-determination), which goes beyond a simple delegation of power. The impact of empowerment in different fields of Psychology has been important due to the proven relations maintained with 
other well-founded psychological variables in the scientific literature, and because of the positive consequences on the results and wellbeing of people. Although there are different instruments for the measurement of this construct, the Psychological Empowerment Instrument (PEI) by Spreitzer (1995) has been traditionally the one most employed to measure the psychological empowerment in the area of Organizational Psychology, counting with more than one thousand publications, and articles as well as translations and adaptations to different languages. The original questionnaire elaborated by Spreitzer consists of 16 items organised in four factors following the four basic cognitions that were described above. Therefore, the aim of this article is the adaptation to Spanish, validation of the PEI, and to analyse its factor structure.

In order to do so, two independent studies were carried out. The specific objective of the first study was to translate into Spanish and to adapt culturally such questionnaire. In the second study, the objective was to validate the resulting questionnaire and to analyse the factor structure and its goodness and with other psychological and organisational variables as well as the external validity in a sample of workers. In order to do so, in the first study it was carried out an inverse translation and adaptation to Spanish with a sample of 242 university students to subsequently analyse the factorial structure of the items and its reliability. The resulting translated questionnaire confirmed the factor structure in four dimensions (Competence, Meaning, Self-determination, and Impact), although the number of items was reduced from the original 16 to 13 , which would explain the $74.08 \%$ of the sampling variance.

To confirm the psychometric characteristics of this new questionnaire, we performed a second study with 317 workers of a Spanish service company in order to analyse through confirmative procedures the internal structure of this new instrument, verify its adequacy and goodness of fit of the resulting factors as well as assessing the relation and external validity in a sample of workers and concurrency with other variables (structural empowerment, affective commitment and intention to leave), which are known to have a relation with psychological empowerment from previous research. From the application of this new questionnaire translated to Spanish into this sample of workers, we confirmed again the structure of 13 items in four factors from the first study. Furthermore, we obtained good fit indexes (GFI, AGF, and NNFI), with values between .90 and .96 . Additionally, through regression analysis we obtained positive and statistically significant results between psychological empowerment and other variables included in the study (structural empowerment, affective commitment, and intention to leave), testing the interrelations among them. Therefore, those people with a greater cognition on their empowerment developed a greater engagement and attachment towards the organisation, and consequently, their wish to leave was lower.

Our study concludes with a discussion about the importance of the adapted instrument and its possible applications.

Keywords: commitment, empowerment, intent to leave, questionnaire validation, Spanish.

\section{Introducción}

El constructo del empoderamiento apareció en el campo de la psicología y, especialmente, su aplicación en el ámbito comunitario, en la década de los ochenta del siglo pasado a través de los trabajos de Julian Rappaport (Miguel et al., 2015), aunque rápidamente ha sido aplicado a otros ámbitos, entre ellos el organizacional (Conger y Kanungo, 1988). En cuanto a la naturaleza de este constructo, mucho se ha debatido sobre su carácter multidimensional, y sobre la existencia y relación de varios componentes cognitivos, emocionales, conductuales o relacionales (Rodrigues et al., 2018).

En el ámbito de la psicología organizacional 
seha enfatizado, especialmente, sucomponente cognitivo, yque se lo conceptualiza como la creencia que tiene un trabajador sobre el control, nivel de autonomía y toma de decisiones acerca de su trabajo (Conger y Kanungo, 1988); lo que le llevará a una conducta más intraemprendedora y activa dentro de la organización (Bretones, 2014), con implicaciones sobre su satisfacción (Li., et al, 2018), bienestar y salud (Jáimez y Bretones, 2011; Laschinger y Read, 2017).

Este tipo de empoderamiento psicológico se diferenciaría de otros tipos como el empoderamiento estructural, el cual había sido previamente caracterizado (Kanter,1993) como aquellas prácticas organizativas que permiten al trabajador alcanzar estructuras de empoderamiento, tales como el acceso a los recursos necesarios para desempeñar una tarea, el acceso a la información necesaria y el apoyo de compañeros y superiores. Diversas investigaciones han comprobado las relaciones entre empoderamiento estructural y psicológico, de manera que este último sería un consecuente del empoderamiento estructural (Kazlauskaite et al., 2012; Zhang et al., 2018).

También el empoderamiento psicológico se ha relacionado con el compromiso organizacional, en la medida que se trata de un estado psicológico que caracteriza la relación de un empleado con su entorno de trabajo (Bohrt y Bretones, 2018). Entre las teorías más relevantes sobre el compromiso organizacional, se puede citar a Meyer y Allen (1997), quienes señalaron que este estaría integrado por tres componentes diferentes: la identificación afectiva del empleado hacia la organización (al que llamaron "compromiso afectivo"), el relativo a los costos (personales y económicos) asociados con dejar la organización (compromiso de continuidad), y la obligación moral de permanecer en la organización (llamado compromiso normativo).

Diversos estudios han confirmado las relaciones entre el compromiso y otras variables cognitivas (Lupano Perugini y Castro Solano, 2018), y entre ellas, el empoderamiento psicológico, que actúa este último como un antecesor del compromiso (Abdulrab et al., 2017) en la medida que lo vincula con el entorno en el que trabaja $y$, especialmente, con el compromiso afectivo (Morin et al., 2016). Finalmente, otra de las variables que se ha relacionado con el empoderamiento psicológico ha sido la de rotación o abandono de la organización.

Tradicionalmente, los modelos explicativos sobre las razones principales por las que los empleados se quedaban o tenían intención de abandonar la organización a la que estaban vinculados se han basado en variables de satisfacción laboral o baja retribución (Bretones y González, 2009; Chang et al., 2013).

Sin embargo, en los últimos años, diversas investigaciones han puesto de relieve la importancia de otros factores relacionados con la intención de irse o permanecer en una organización, como ha sido el empoderamiento psicológico (Ouyang et al., 2020). Varios estudios han comprobado una relación directa de este en la reducción tanto de la intención de rotación como de la rotación real (Gardner et al., 2011; Kim y Fernández, 2017), por lo que se demostraría que el empoderamiento psicológico es un fuerte antecedente de la rotación.

\section{Medidas del empoderamiento psicológico}

Varios son los instrumentos de medida que, desde distintas áreas de la psicología, se han desarrollado para la evaluación del empoderamiento psicológico (Miguel, Ornelas, y Marôco, 2015), aunque cada uno de ellos ha estado influenciado por una conceptualización o modelo teórico de empoderamiento psicológico.

En el área de la psicología organizacional, uno de los primeros instrumentos fue el Psychological Empowerment Instrument (PEI), desarrollado por Spreitzer (1995). El PEI es una 
medida multidimensional de empoderamiento psicológico aplicable en contextos laborales que se basa en el modelo de empoderamiento de Thomas y Velthouse (1990). Estos autores establecieron que el empoderamiento psicológico estaba compuesto por cuatro cogniciones básicas (impacto, competencia, significado y autonomía), algunas de las cuales serían consideradas después como necesidades básicas dentro de la teoría de la autodeterminación (Self-Determination Theory, SDT) de Deci y Ryan (2000). El PEI de Spreitzer (1995) tenía una estructura factorial en cuatro dimensiones, una por cada cognición, conformada por tres ítems cada una. En un trabajo posterior (Spreitzer, 1996) incluyó un ítem más para cada una de estas dimensiones, por lo que el instrumento final está conformado por cuatro subescalas de cuatro ítems cada una. Desde entonces, el PEI de Spreitzer $(1995,1996)$ ha sido el más empleado en el campo de la psicología organizacional, y es utilizado en más de mil publicaciones científicas (Seibert et al., 2011), especialmente, en el sector sanitario (Li et al., 2018), aunque también se ha utilizado para medir el nivel de empoderamiento psicológico en trabajadores de otros sectores de actividad (Ayala Calvo y García, 2018). En todos estos casos, el cuestionario ha dado muy buenos resultados psicométricos. Además, aunque este instrumento fue creado originalmente en inglés, existen diferentes traducciones y adaptaciones posteriores a diversas lenguas como el español (Albar et al., 2012), portugués (Santos et al., 2014; Schumaher et al., 2019), chino (Sun et al., 2011) y turco (Uner y Turan, 2010).

Por todo ello, el objetivo general de esta investigación ha sido la traducción al español y la validación del Psychological Empowerment Instrument (PEI), analizando su estructura multidimensional. Se llevaron a cabo dos estudios independientes: el primero, con el objetivo específico de traducir y adaptar culturalmente el cuestionario proponiendo una estructura factorial; el segundo, con el objeto de comprobar las características psicométricas, confirmar la estructura factorial obtenida y comprobar la bondad del nuevo modelo, a partir de evaluar la relación con otras variables psicológicas y organizativas, y la validez externa en otra muestra de trabajadores.

\section{Estudio 1: Traducción}

\section{y estructura factorial de la Escala}

\section{de Empoderamiento Psicológico}

\section{Método}

\section{Participantes}

En el estudio 1 participaron 242 estudiantes de la Facultad de Relaciones Laborales y Recursos Humanos de la Universidad de Granada en un rango de edad entre 19 y 58 años, con un promedio de 25.92 años. Dos de cada tres participantes eran mujeres. Los criterios de inclusión para participar en el estudio fueron tener más de 18 años y tener como lengua materna el idioma español. La participación era anónima y voluntaria, sin recibir los participantes ningún tipo de incentivo. La muestra fue recogida mediante muestreo accidental no probabilístico, y su tamaño resultó adecuado para el propósito del estudio (McNeish, 2017; Robinson, 2018).

\section{Instrumentos}

El PEI (Spreitzer, 1995, 1996) está compuesto por cuatro subescalas (competencia, significado, autonomía e impacto) de cuatro ítems cada una, en una escala de respuestas que va desde 1 = totalmente en desacuerdo a 7 = totalmente de acuerdo.

\section{Procedimiento}

Para el proceso de traducción y adaptación se siguió el procedimiento de Hambleton y Zeniski (2011). Así, el primer paso fue una 
traducción inversa de los 16 ítems originales del cuestionario al español. Para ello, se contó con la colaboración de cuatro profesores universitarios del área de psicología que no participaban en el estudio, quienes realizaron la traducción del cuestionario del inglés al español de manera independiente. A continuación, se compararon las cuatro traducciones y se debatieron con ellos las diferencias existentes hasta llegar a un consenso en cada uno de los ítems, con el fin de obtener una sola versión en español de los distintos ítems.

El siguiente paso fue realizar la traducción de la versión española obtenida del cuestionario al inglés. Dicha traducción fue realizada por una traductora profesional totalmente ajena a la primera traducción y cuyo idioma materno era el inglés. Posteriormente, se compararon las dos versiones inglesas, la original y la obtenida de la traducción de la versión española, para analizar la bondad de la traducción en función del grado de coincidencia de los ítems en ambos cuestionarios y modificar aquellos ítems que así lo requirieran.

Para analizar la validez de la escala en español construida, se sometió cada uno de los ítems a evaluación mediante juicio de expertos. Para ello, se contó con la participación de tres expertos (dos de ellos en el constructo a evaluar y otro en la construcción de escalas).

Para poder llevar a cabo la evaluación de manera efectiva, se les facilitó el concepto de empoderamiento psicológico y el de cada una de las dimensiones que lo componen. Posteriormente, se les dio un listado con todos los ítems, de tal forma que la tarea de los jueces consistió en clasificar cada uno de los ítems en aquella dimensión en la que ellos consideraran que pertenecía. También se les ordenó que expresaran su opinión sobre si el número de ítems por dimensión era suficiente para medir cada una de las dimensiones. Por último, se les pidió que evaluasen si los ítems estaban redactados de forma clara (International Test
Commission. 2017).

De dicho juicio de expertos, se obtuvieron unos resultados muy favorables, ya que los tres jueces clasificaron de manera adecuada cada uno de los ítems en la dimensión correspondiente, y coincidieron también en que las dimensiones podían ser perfectamente medidas con cuatro ítems. No obstante, se realizó una redacción más clara de algunos de los ítems.

El resultado de todas las fases anteriores fue la traducción al español del PEI, compuesta por un total de 16 ítems y cuatro dimensiones: competencia, significado, autonomía e impacto. Las respuestas fueron recogidas en una escala tipo Likert del 1 al 7, en la que 1 equivale a poco y 7 a demasiado. $\mathrm{Al}$ igual que en el cuestionario original, la puntuación total de empoderamiento psicológico será la puntuación media de la suma de los ítems, de manera que, a mayor puntuación media, mayor es el nivel de empoderamiento psicológico.

Obtenida la traducción española del PEI, se procedió a la recogida de datos y posterior análisis cuantitativo de los ítems para su análisis factorial (Goretzko et al., 2019).

\section{Análisis de los datos}

Los datos obtenidos fueron analizados mediante estadísticos descriptivos (media y desviación típica) y correlacionales mediante el programa informático SPSS(c) (versión 20.0. IBM Company, Chicago, IL). En cuanto al método de extracción de factores, se utilizó el análisis de componentes principales y la rotación Varimax (Kline, 2013).

\section{Resultados}

Recogidos los datos, se realizaron unos primeros análisis descriptivos (media y desviación típica de cada ítem, coeficiente de correlación corregido entre la puntuación en el ítem y la total obtenida en la dimensión a la que pertenece), y de fiabilidad de cada una de las dimensiones propuestas en el cuestionario 
original, así como la fiabilidad de cada dimensión si el ítem es eliminado. En este sentido, se tomaron como criterios de adecuación que la desviación típica por ítem fuera superior a 1 , su coeficiente de correlación corregido entre la puntuación del ítem y la dimensión fuera superior a .30 y que la fiabilidad de la dimensión no aumentara cuando el ítem fuera eliminado (International Test Commission, 2017).
Como se puede observar en la Tabla 1, todos los ítems obtuvieron una desviación típica superior a 1 (a excepción del ítem 1 que fue cercana, del .91). Por otro lado, todos los ítems tuvieron unas correlaciones con sus dimensiones originarias superiores a .30 . Aun así, debemos indicar que los ítems 8 y 12 mostraron una correlación negativa con su dimensión de origen.

\section{Tabla 1}

Media y desviación típica de cada item; coeficiente de correlación corregido.

\begin{tabular}{cccc}
\hline Ítems & M & DT & R IT-c \\
\hline $\begin{array}{c}\text { Empoderamiento psicológico } \\
\text { Competencia }\end{array}$ & & & \\
$\begin{array}{c}\text { 1. Tengo confianza en mi capacidad para hacer mi } \\
\text { trabajo. }\end{array}$ & 6.25 & .91 & .72 \\
$\begin{array}{c}\text { 9. Mi trabajo está acorde con mis habilidades/ } \\
\text { capacidades. }\end{array}$ & 3.76 & 2.04 & .53 \\
$\begin{array}{c}\text { 11. He adquirido las habilidades necesarias para mi } \\
\text { trabajo. }\end{array}$ & 5.57 & 1.46 & .52 \\
$\begin{array}{c}\text { 15. Estoy seguro de mí mismo/a acerca de mis } \\
\text { capacidades para realizar las actividades de mi trabajo. }\end{array}$ & 6.04 & 1.09 & .66 \\
\hline
\end{tabular}

\section{Significado}

2. El trabajo que hago es importante para mí.

$\begin{array}{lll}4.95 & 1.87 \quad .84\end{array}$

5. Las actividades laborales tienen un significado personal para mí.

$\begin{array}{lll}4.82 & 1.81 \quad .77\end{array}$

8. Realmente me importa lo que hago en mi trabajo. $\begin{array}{llll}2.64 & 1.65 & -.51\end{array}$
13. El trabajo que hago tiene sentido para mí.
$4.81 \quad 1.80$
.85

\section{Autonomía}

3. Tengo bastante autonomía para decidir cómo hacer mi trabajo.

$\begin{array}{lll}4.51 & 1.75 \quad .86\end{array}$

7. Puedo decidir por mí mismo/a cómo voy a realizar mi propio trabajo.

$4.37 \quad 1.88 \quad .90$

$\begin{array}{lll}4.19 & 1.96 \quad .87\end{array}$

libertad en la forma de hacer mi trabajo.

16. Tengo la oportunidad de utilizar la iniciativa personal para llevar a cabo mi trabajo.

$\begin{array}{lll}4.54 & 1.81 \quad .81\end{array}$




\begin{tabular}{cllll}
\hline Ítems & M & DT & R IT-c \\
\hline Empoderamiento psicológico & & & \\
\hline
\end{tabular}

Impacto

4. Mi influencia sobre lo que ocurre en mi departamento o área es alta.

6. Tengo mucho control sobre lo que sucede en mi departamento.

12. Mi opinión cuenta en la toma de decisiones del departamento o área.

14. Tengo bastante influencia sobre lo que ocurre en mi departamento.

\section{$\begin{array}{lll}3.95 & 1.96 \quad .90\end{array}$}

$\begin{array}{lll}3.63 & 1.97 & .89\end{array}$

$\begin{array}{lll}4.39 & 1.99 & -.49\end{array}$

$\begin{array}{lll}3.54 & 2.03 \quad .85\end{array}$
En cuanto a los análisis de fiabilidad, se observa que la única dimensión que tuvo una fiabilidad aceptable, manteniendo la composición de ítems original, fue la que se refiere a la autonomía $(\alpha=.88)$. Las demás dimensiones mostraron bajos índices de fiabilidad $(\alpha$ $=.27$ para competencia; $\alpha=.08$ para significado, y $\alpha=.19$ para impacto). Sin embargo, realizados los análisis de fiabilidad de cada una de las dimensiones, se comprobó que en la de competencia, al eliminar el ítem 9 ("Mi trabajo está acorde con mis habilidades/capacidades") su fiabilidad aumentaba a .70. Del mismo modo, en la dimensión de significado, eliminando el ítem 8 ("Realmente me importa lo que hago en mi trabajo"), la fiabilidad ascendía a .83. Asimismo, en la dimensión de impacto, suprimiendo el ítem 12 ("Mi opinión cuenta en la toma de decisiones de mi departamento o área"), la fiabilidad se elevaba a .92.
Por último, para confirmar la conveniencia o no de eliminar estos ítems, se decidió realizar un análisis de fiabilidad de la escala de empoderamiento psicológico en su conjunto eliminando los ítems 8,9 y 12 . lo que resultóEl resultado fue que, de este modo, la fiabilidad global del cuestionario aumentaba de $\alpha=.60$ a $\alpha=.89$. Por ello, se tomó la decisión de continuar los análisis con la eliminación de los tres ítems descriptos anteriormente.

A continuación, para estudiar la homogeneidad de las dimensiones, se realizó un análisis de correlación entre las puntuaciones de cada uno de los ítems y la puntuación total de las dimensiones. En este caso, el criterio para considerar un ítem adecuado fue que la diferencia entre la correlación obtenida con la dimensión a la que pertenece y la correlación obtenida con las demás dimensiones debía ser superior a .20 (Jackson, 1970).

\section{Tabla 2}

Correlaciones entre las puntuaciones de cada uno de los ítems y su dimensión.

\section{Ítem Competencia Significado Autonomía Impacto}

\section{Competencia}

1. Tengo confianza en mi capacidad para hacer mi trabajo.

11. He adquirido las habilidades necesarias para mi trabajo. 


\section{Ítem}

Competencia Significado Autonomía Impacto

15. Estoy seguro de mí mismo/a acerca

de mis capacidades para realizar las

actividades de mi trabajo.

$\begin{array}{lll}.66 & .05 & .22\end{array}$

.07

\section{Significado}

2. El trabajo que hago es importante para

$$
\text { mí. }
$$

$-.23$

.84

.42

5. Las actividades laborales tienen un significado personal para mí.

$-.26$

.77

.33

13. El trabajo que hago tiene sentido para mí.

$-.24$

.85

.42

.31

\section{Autonomía}

3. Tengo bastante autonomía para decidir cómo hacer mi trabajo.

$-.05$

.34

.86

7. Puedo decidir por mí mismo/a cómo voy a realizar mi propio trabajo.

$\begin{array}{lllll}.07 & .35 & .87 & .52\end{array}$

10. Tengo bastantes oportunidades de independencia y libertad en la forma de hacer mi trabajo.

16. Tengo la oportunidad de utilizar la

$-.05$

.39

.87

.52

iniciativa personal para llevar a cabo mi

.00

.41

.81 trabajo.

\section{Impacto}

4. Mi influencia sobre lo que ocurre en mi departamento o área es alta.

$-.12$

.44

.55

.90

6. Tengo mucho control sobre lo que sucede en mi departamento.

$-.12$

.45

.56

14. Tengo bastante influencia sobre lo que ocurre en mi departamento.

$-.09$

.50

.63

.85

Del análisis de los datos, se observa que todos los ítems obtuvieron un índice de correlación mucho mayor con la dimensión originaria de pertenencia que con el resto de dimensiones, de manera que se cumplió el criterio establecido por Jackson (1970).

Finalmente, para conocer la estructura interna de la versión española del PEI, se llevó a cabo un análisis factorial exploratorio sobre los 13 ítems (Goretzko, Pham y Bühner, 2019). El método de extracción de factores utilizado fue el análisis de componentes principales y el tipo de rotación Varimax. De los resultados obtenidos, se observó que el cuestionario traducido y adaptado posee una estructura multidimensional en cuatro factores constituidos por tres ítems cada uno claramente diferenciados (véase Tabla 3). 
Tabla 3

Análisis factorial exploratorio.

\begin{tabular}{|c|c|c|c|c|}
\hline Factor & F1 & F2 & F3 & F4 \\
\hline 1. Tengo confianza en mi capacidad para hacer mi trabajo. & -.05 & .05 & -.04 & .89 \\
\hline $\begin{array}{l}\text { 11. He adquirido las habilidades necesarias para mi } \\
\text { trabajo. }\end{array}$ & .15 & .04 & .40 & .55 \\
\hline $\begin{array}{l}\text { 15. Estoy seguro de mí mismo/a acerca de mis } \\
\text { capacidades para realizar las actividades de mi trabajo. }\end{array}$ & .02 & .04 & .11 & .87 \\
\hline 2. El trabajo que hago es importante para mí. & .80 & .21 & .17 & .09 \\
\hline $\begin{array}{l}\text { 5. Las actividades laborales tienen un significado personal } \\
\text { para mí. }\end{array}$ & .75 & .27 & .06 & -.08 \\
\hline 13. El trabajo que hago tiene sentido para mí. & .83 & .17 & .18 & .08 \\
\hline $\begin{array}{l}\text { 3. Tengo bastante autonomía para decidir cómo hacer mi } \\
\text { trabajo. }\end{array}$ & .16 & .26 & .81 & -.00 \\
\hline $\begin{array}{l}\text { 7. Puedo decidir por mí mismo/a cómo voy a realizar mi } \\
\text { propio trabajo. }\end{array}$ & .16 & .25 & .82 & .16 \\
\hline $\begin{array}{l}\text { 10. Tengo bastantes oportunidades de independencia y } \\
\text { libertad en la forma de hacer mi trabajo. }\end{array}$ & .22 & .29 & .80 & .05 \\
\hline $\begin{array}{l}\text { 16. Tengo la oportunidad de utilizar la iniciativa personal } \\
\text { para llevar a cabo mi trabajo. }\end{array}$ & .23 & .35 & .66 & .16 \\
\hline $\begin{array}{l}\text { 4. Mi influencia sobre lo que ocurre en mi departamento o } \\
\text { área es alta. }\end{array}$ & .17 & .85 & .24 & .02 \\
\hline $\begin{array}{l}\text { 6. Tengo mucho control sobre lo que sucede en mi } \\
\text { departamento. }\end{array}$ & .20 & .85 & .26 & .00 \\
\hline $\begin{array}{l}\text { 14. Tengo bastante influencia sobre lo que ocurre en mi } \\
\text { departamento. }\end{array}$ & .29 & .83 & .32 & .08 \\
\hline$\%$ Varianza explicada & 44.01 & 12.87 & 10.36 & 6.82 \\
\hline Autovalor & 7.04 & 2.06 & 1.65 & 1.09 \\
\hline Alfa de Cronbach & .83 & .92 & .88 & .70 \\
\hline
\end{tabular}

En resumen, el nuevo cuestionario traducido estaría conformado por un total de cuatro dimensiones al igual que en su escala original (competencia, significado, autonomía e impacto), compuestas cada una por tres ítems, a excepción de la dimensión de autonomía, que estaría formada por cuatro ítems que explican el $74.08 \%$ de la varianza de la muestra. De los cuatro factores, el factor 1 (F1, significado) explicaría un mayor porcen- taje de varianza $(\mathrm{F} 1=44.01 \%)$ con un índice de fiabilidad $\alpha=.83$. El factor 2 (F2, impacto) explicaría un $12.87 \%$ de la varianza con una consistencia interna de $\alpha=.92$. El tercer factor (F3, autonomía) presentaba una varianza de $10.36 \%$ y una fiabilidad de $\alpha=.88$. El cuarto y último factor (F4, competencia) explicaría un $6.82 \%$ de la varianza y tenía una consistencia interna más modesta, de $\alpha=.70$. 


\section{Estudio 2: Análisis factorial}

\section{confirmatorio y validez concurrente}

\section{del PEI en el ámbito laboral}

\section{Método}

Se decidió realizar un segundo estudio independiente en el cual se pudiera comprobar con procedimientos confirmatorios la estructura interna del instrumento obtenido en el anterior, además de analizar su validez concurrente y externa en una muestra de trabajadores.

\section{Participantes}

La muestra de este estudio estuvo conformada por 317 trabajadores de una empresa española del sector servicios que opera a nivel nacional, se realizó mediante la técnica de muestreo accidental no probabilístico. Con el propósito de aislar la variable sexo de la muestra, se intentó balancear el número de mujeres $(51.5 \%)$ y hombres $(48.5 \%)$ participantes. La edad promedio de esta muestra fue de 34.39 años con un rango de entre 17 y 64 años, y un promedio de antigüedad en la empresa de 5.66 años. Los participantes en el estudio lo hicieron de manera anónima y voluntaria, sin recibir ningún tipo de incentivo por su participación.

\section{Instrumentos}

Los instrumentos de medida utilizados en nuestro segundo estudio fueron los siguientes:

1) Empoderamiento psicológico: para medir esta variable se empleó la traducción al español del PEI derivada del estudio 1, conformado por 13 ítems en las cuatro dimensiones anteriormente descriptas.

También se comprobó la validez concurrente del nuevo cuestionario con otras variables psicológicas y organizativas, las cuales habían sido ampliamente estudiadas en su relación con el empoderamiento psicológico (Seibert et al., 2011).

2) Empoderamiento estructural: para medirlo utilizamos el Conditions of Work Effectiveness Questionnaire CWEQ (Laschinger et al., 2004) en su adaptación y validación al español de Jáimez y Bretones (2013).

En cuanto a las variables consecuentes, tal como se señaló en la introducción, se tomaron en cuenta otras dos variables: el compromiso afectivo, que para medirla se utilizó las Commitment Scales de Meyer y Allen (1997) a través de la adaptación al español elaborada por Arciniega y González (2006). Dicha versión consta de seis ítems en una sola dimensión. La otra variable fue intención de irse y para su análisis se elaboraron tres ítems en escala tipo Likert: $1=$ a menudo pienso dejar el trabajo, 2 = yo buscaré un nuevo trabajo probablemente en el próximo año, y 3 = cuando pueda me iré de esta empresa. Estos fueron intercalados entre los ítems de las escalas de compromiso organizacional.

\section{Resultados}

En primer lugar, con la intención de confirmar los resultados obtenidos en el estudio 1, se llevó a cabo un análisis estadístico de los ítems obtenidos en esta nueva muestra. Los datos descriptivos (media, desviación típica y rango de respuesta) de esta segunda muestra confirmarían los conseguidos en el estudio 1. Los índices de correlación ítemtotal corregidos resultaron adecuados para todas las subescalas, de ahí que los valores de la dimensión de competencia estuvieron entre $\mathrm{r}=.74$ y $\mathrm{r}=.82$; los de la dimensión significado entre $\mathrm{r}=.84 \mathrm{y} \mathrm{r}=.91$; los valores de autonomía entre $\mathrm{r}=.56$ y $\mathrm{r}=.60$; y los valores de la dimensión de impacto entre $r=$ $.86 \mathrm{y} \mathrm{r}=.90$.

Seguidamente, se realizó un análisis factorial exploratorio, por el método de extracción de componentes principales 
y posterior rotación Varimax. Los datos obtenidos confirmaron la estructura de cuatro factores obtenida en el estudio 1. Un primer factor (autonomía) que explicaría el $45.94 \%$ de la varianza; un segundo factor (impacto) con un $12.69 \%$ de la varianza; un tercer factor (significado) que explicaría el $8.05 \%$ de la varianza, $y$, por último, un cuarto factor (competencia) con un $7.12 \%$ de la varianza.

Una vez confirmados los resultados obtenidos en el estudio 1, y con el fin de confirmar la estructura empírica de la escala, se realizó un análisis factorial confirmatorio. El modelo a comprobar estaría formado por los cuatro factores de tres ítems cada uno a excepción del factor de autonomía, compuesto por cuatro ítems tal como se propuso en el estudio 1. Para evaluar la adecuación del modelo, se calculó el índice de chi-cuadrado, el cual resultó estadísticamente significativo $\left(\chi^{2}=328.83, \mathrm{p}<.01\right)$. A pesar de ello, se decidió llevar a cabo diversos análisis de bondad de ajuste del modelo, ya que el análisis del chi-cuadrado es susceptible a variaciones en función del tamaño de la muestra.

Los resultados obtenidos mostraron buenos índices de ajuste con valores superiores a .85
(Brown y Cudeck, 1993; Schermelleh-Engel et al., 2003) tanto en el índice de bondad de ajuste $(\mathrm{GFI}=.96)$ como en el índice ajustado de bondad de ajuste (AGFI $=.94)$ e índice de ajuste no normado $(\mathrm{NNFI}=.90)$. Por tanto, estos resultados vienen a corroborar la estructura interna de la versión española del PEI de Spreitzer (1995, 1996), tal como aparece en el Anexo.

En cuanto a las evidencias, se realizó un análisis de validez de criterio entre la variable empoderamiento psicológico $\mathrm{y}$ sus dimensiones, con las variables empoderamiento estructural, compromiso afectivo e intención de irse, por su relación, tal como reflejaba la literatura existente. Para ello, se hizo, en primer lugar, un análisis correlacional y posteriormente, varios análisis de regresión para comprobar dicha predicción.

En la Tabla 4, se observa que tanto el empoderamiento psicológico como cada una de sus dimensiones correlacionan significativamente y en sentido positivo con el compromiso afectivo y con el empoderamiento estructural. Con la intención de irse también poseen una correlación significativa, aunque de signo negativo.

Tabla 4

Análisis de intercorrelaciones de las variables de estudio.

\begin{tabular}{|c|c|c|c|c|c|c|c|}
\hline & 1 & 2 & 3 & 4 & 5 & 6 & 7 \\
\hline \multicolumn{8}{|l|}{ 1. E. psicológico } \\
\hline 2. Competencia & $.53 * *$ & & & & & & \\
\hline 3. Significado & $.82 * *$ & $.41 * *$ & & & & & \\
\hline 4. Autonomía & $.88 * *$ & $.33 * *$ & $.58 * *$ & & & & \\
\hline 5. Impacto & $.85^{* *}$ & $.27 * *$ & $.59 * *$ & $.65 * *$ & & & \\
\hline 6. Emp. estructural & $.57 * *$ & $.24 * *$ & $.47 * *$ & $.52 * *$ & $.45^{* *}$ & & \\
\hline 7. Comp. afectivo & $.66^{* *}$ & $.24 * *$ & $.63 * *$ & $.58 * *$ & $.52 * *$ & $.69 * *$ & \\
\hline 8. Intención de irse & $.40 * *$ & $.19^{* *}$ & -.46 & $.32 * *$ & $.27 * *$ & $.48 * *$ & $.65 * *$ \\
\hline
\end{tabular}

Notas: $* \mathrm{p}<.05 ; * * \mathrm{p}<.01$.

Asimismo, realizados los análisis de regresión, se pudo comprobar que el empoderamiento psicológico tiene una relación significativa con el empoderamiento 
estructural $(\beta=.57 \mathrm{p}=.00)$, el compromiso afectivo $(\beta=.66 \mathrm{p}=.00)$ y el nivel de intención de irse de los trabajadores $(\beta=$ $-.40 \mathrm{p}=.00)$. También se realizó el análisis de regresión entre las diferentes subescalas (competencia, significado, autonomía e impacto) y el empoderamiento estructural, el compromiso afectivo y la intención de irse de los trabajadores. Realizados dichos análisis, se comprobó que el empoderamiento estructural tenía una relación estadísticamente significativa mediante pruebas asociativas de regresión con cada una de las cuatro dimensiones del PEI (competencia: $\beta=.24, p$ $=.00$; significado: $\beta=.47, \mathrm{p}=.00$; autonomía: $\beta=.52, p=.00$; impacto: $\beta=.45, \mathrm{p}=.00$ ). Por otra parte, estas dimensiones serían buenos antecesores del compromiso afectivo (competencia: $\beta=.24, p=.00$; significado: $\beta=.63, p=.00$; autonomía: $\beta=.58, \mathrm{p}=$ .00 ; impacto: $\beta=.52, \mathrm{p}=.00$ ); así como de la intención de irse de los trabajadores (competencia: $\beta=-.19, p=.00$; significado: $\beta$ $=-.46, \mathrm{p}=.00$; autonomía: $\beta=-.32, \mathrm{p}=.00$; impacto: $\beta=-.28, p=.00$ ).

\section{Discusión}

El deseo de contar con instrumentos confiables, válidos y parsimoniosos ha sido un objetivo central de la Psicología en todos sus ámbitos, incluido el organizacional. En este caso, el objetivo de este estudio era la traducción al español y validación del PEI (Spreitzer, 1995, 1996). Realizadas todas las fases de traducción inversa del cuestionario original, así como los análisis estadísticos pertinentes, se debe indicar que la versión al castellano del instrumento propuesto en este trabajo (véase el Anexo) queda perfectamente validada, ya que tanto las puntuaciones obtenidas en los análisis factorial y de consistencia interna demuestran que el ajuste del modelo factorial es adecuado.

Este estudio ha permitido comprobar que el PEI tiene un carácter multidimensional constituido por cuatro subescalas (compe- tencia, significado, autonomía e impacto) en la misma línea que otros estudios (Seibert et al., 2011), aunque, en el caso de la adaptación que se presenta (véase el Anexo), el número de ítems de cada una de las subescalas varía con respecto al cuestionario original, de manera que las subescalas estarían compuestas por tres ítems: competencia (ítems 1, 11 y 15), significado (ítems 2, 5 y 13) e impacto (ítems 4,6 y 14), mientras que la subescala autonomía, por cuatro (ítems $3,7,10$ y 16 ), la cual se acerca más al formato original de Spreitzer de 1995 que la modificación que hizo la autora posteriormente.

En este estudio, también se puede observar la validez concurrente o aproximación del cuestionario adaptado con otros, cuya relación ha sido comprobada previamente en la literatura. En este sentido, la validación al español que se presenta aporta novedad sobre otras adaptaciones y validaciones en otros idiomas. Los datos obtenidos en este estudio muestran relaciones estadísticamente significativas de cada una de las cuatro dimensiones con los constructos que han reportado relaciones en estudios previos, como han sido el empoderamiento estructural (Kazlauskaite et al., 2012; Zhang et al., 2018), el compromiso afectivo (Morin et al., 2016) y la rotación (Gardner et al., 2011; Kim y Fernández, 2017). Así, se pudo comprobar que el empoderamiento psicológico está antecedido por las prácticas organizativas (empoderamiento estructural) y que tiene una estrecha vinculación con el compromiso afectivo, de manera que aquellas personas con una mayor cognición acerca de su empoderamiento desarrollan una mayor vinculación y adhesión hacia la organización y el centro de trabajo y, por tanto, un menor deseo de abandonarla.

Como todo estudio, este tiene limitaciones. Uno de ellos es que la generalidad de los hallazgos está limitada por el uso de una muestra de conveniencia, en este caso en una empresa del sector servicios en España, por lo que sería conveniente realizar nuevos 
estudios que permitan verificar la adaptación del PEI en otros sectores y entornos culturales. También sería recomendable estudiar la validez concurrente con otros constructos análogos, tales como la autoeficacia o el locus de control.

Se considera, por último, que esta validación del PEI al español provee de una útil e importante herramienta para los investigadores en el estudio del empoderamiento psicológico en el ámbito laboral y permite avanzar en el estudio de esta variable psicológica y su relación con distintas variables psicológicas en otras muestras de trabajadores.

\section{Directrices éticas}

Todos los procedimientos realizados en ambos estudios siguieron los estándares éticos internacionales de la declaración de Helsinki de 1964 y sus posteriores modificaciones, y de la Asociación Americana de Psicología (APA). Todos los participantes fueron voluntarios, no recibieron compensación alguna $\mathrm{y}$ manifestaron su autorización a participar en el estudio.

\section{Referencias}

Abdulrab, M., Zumrah, A. R., Almaamari, Q. y Altahitah, A. (2017). The role of psychological empowerment on work engagement: The development of conceptual framework. International Journal of Business Management and Economic Research, 8(6), 1157-1163.

Albar, M. J., García-Ramírez, M., Jiménez, A. M. L y Garrido, R. (2012). Spanish adaptation of the scale of psychological empowerment in the workplace. The Spanish Journal of Psychology, 15(2), 793-800. https://doi.org/10.5209/rev_ sjop.2012.v15.n2.38891

Arciniega, L. M. y González, L. (2006). What is the influence of work values relative to other variables in the development or organizational commitment? Revista de Psicología Social, 21, 35-50. https://doi. org/10.1174/021347406775322269.
Ayala Calvo, J. C. y García, G. M. (2018). Hardiness as moderator of the relationship between structural and psychological empowerment on burnout in middle managers. Journal of Occupational and Organizational Psychology, 91(2), 362-384. https://doi.org/10.1111/ joop. 12194.

Bohrt, R. y Bretones, F. D. (2018). El compromiso organizacional y su relación con el intercambio líder-empleado y la satisfacción laboral. Revista de Trabajo y Seguridad Social, 419, 187-212.

Bretones, F. D. (2014). Entrepreneurial employees. En A. Manuti y P. Davide de Palma (Eds.) Why human capital is important for organizations: People come first (pp. 53-61). Londres: Palgrave McMillan.

Bretones, F. D. y González, J. M. (2009). Absentismo y rotación laboral. En V. Zarco y J. M. González (Eds.), Psicología del Trabajo (pp. 91-113). Madrid: Pirámide.

Brown, M. W. y Cudeck, R., (1993). Alternative ways of assessing model fit. En K. Bollen y L. Long (Eds.), Testing Structural Equation Models (pp. 136-162). Newbury Park, CA: Sage.

Chang, W. J. A., Wang, Y. S. y Huang, T. C. (2013). Work design-related antecedents of turnover intention: A multilevel approach. Human Resource Management, 52(1), 1-26. https://doi.org/10.1002/hrm.21515

Conger, J. A. y Kanungo, R. N. (1988). The empowerment process: Integrating theory and practice. Academy of Management Review, 13, 471-483. https://doi.org/10.5465/ amr.1988.4306983.

Deci, E. L. y Ryan, R. M. (2000). The "what" and "why" of goal pursuits: Human needs and the self-determination of behavior. Psychological Inquiry, 11(4), 227-268. https://doi. org/10.1207/s15327965pli1104_01.

Gardner, T. M., Wright, P. M. y Moynihan, L. M. (2011). The impact of motivation, empowerment, and skill-enhancing practices on aggregate voluntary turnover: The mediating effect of collective affective commitment. Personnel Psychology, 64(2), 315-350. https://doi. 
org/10.1111/j.1744-6570.2011.01212.x

Goretzko, D., Pham, T. T. H. y Bühner, M. (2019). Exploratory factor analysis: Current use, methodological developments and recommendations for good practice. Current Psychology, 1-12. https://doi.org/10.1007/s12144-01900300-2

Hambleton, R. K. y Zeniski, A. L. (2011). Traslating and adapting educational tests for cross-cultural assessments. En D. Matsumoto y F. J. R. van de Vijver (Eds.). Cross-cultural research methods in Psychology (pp 46-70). New York: Cambridge University Press.

International Test Commission. (2017). The ITC Guidelines for Translating and Adapting Tests (Second edition). www.InTestCom.org

Jackson, D. N. (1970). A sequential system for personality scale development. En C. D. Spielberger (Ed.), Current topics in clinical and community psychology (pp. 61-96). Nueva York: Academic Press. https://doi.org/10.1016/ b978-0-12-153502-5.50008-4.

Jáimez, M. J. y Bretones, F. D. (2011). Towards a healthy organisation model: the relevance of empowerment. Is-Guc, The Journal of Industrial Relations \& Human Resource, 13(3), 7-26. https://doi.org/10.4026/1303-2860.2011.0180.x

Jáimez, M. J. y Bretones, F. D. (2013). Spanish adaptation of Structural Empowerment Scale. Spanish Journal of Psychology, 16, 1-7. https:// doi.org/10.1017/sjp.2013.14

Kanter, R. M. (1993). Men and women of the corporation. Nueva York: Basic Books.

Kazlauskaite, R., Buciuniene, I. y Turauskas, L. (2012). Organisational and psychological empowerment in the HRM-performance linkage. Employee Relations, 34(2), 138-158. https://doi.org/10.1108/01425451211191869

Kim, S. Y. y Fernandez, S. (2017). Employee Empowerment and Turnover Intention in the U.S. Federal Bureaucracy. American Review of Public Administration, 47(1), 4-22. https://doi. org/10.1177/0275074015583712.

Kline, R. B. (2013). Exploratory and confirmatory factor analysis. En Y. Petscher y C. Schatsschneider (Eds.), Applied quantitative analysis in the social sciences (pp. 171-207).
Nueva York: Routledge.

Laschinger H., Finegan J. E., Shamian J. y Wilk P. (2004) Longitudinal analysis of the impact of workplace empowerment on work satisfaction. Journal of Organizational Behavior, 25, 527-544. https://doi.org/10.1002/job.256

Laschinger, H. S. y Read, E. (2017). Workplace empowerment and employee health and wellbeing. En C. L. Cooper y M. P. Leiter (Eds.), The Routledge Companion to Wellbeing at Work (pp. 182-196). Londres: Routledge.

Li, H., Shi, Y., Li, Y., Xing, Z., Wang, S., Ying, J., ... y Sun, J. (2018). Relationship between nurse psychological empowerment and job satisfaction: A systematic review and meta-analysis. Journal of Advanced Nursing, 74(6), 12641277. https://doi.org/10.1111/jan.13549

McNeish, D. (2017). Exploratory factor analysis with small samples and missing data. Journal of Personality Assessment, 99(6), 637-652. https://doi.org/10.1080/00223891.2016.1252 382

Meyer, J. P. y Allen, N. J. (1997). Commitment in the workplace: Theory, Research, and Application. Thousand Oaks, CA: Sage.

Miguel, M. C., Ornelas, J. H. y Marôco, P. (2015). Defining psychological empowerment construct: Analysis of three empowerment scales. Journal of Community Psychology, 43(7), 900-919. https://doi.org/10.1002/ jcop. 21721

Morin, A. J., Meyer, J. P., Bélanger, É., Boudrias, J. S., Gagné, M. y Parker, P. D. (2016). Longitudinal associations between employees' beliefs about the quality of the change management process, affective commitment to change and psychological empowerment. Human Relations, 69(3), 839-867. https://doi. org.10.1177/0018726715602046

Ouyang, C., Zhu, Y. y Guo, M. (2020). Effect of empowering leadership on the turnover intention of industrial workers. Social Behavior and Personality: An International Journal, 48(8), 1-11. https://doi.org/10.2224/sbp.9124

Lupano Perugini, M. L. y Castro Solano, A. (2018). Influencia de virtudes organizacionales sobre satisfacción, compromiso y perfor- 
mance laboral en organizaciones argentinas. Interdisciplinaria, 35(1), 171-188. https://doi. org/10.16888/interd.2018.35.1.9

Robinson, M. A. (2018). Using multi-item psychometric scales for research and practice in human resource management. Human Resource Management, 57(3), 739-750. https:// doi.org/10.1002/hrm.21852

Rodrigues, M., Menezes, I. y Ferreira, P. D. (2018). Validating the formative nature of psychological empowerment construct: Testing cognitive, emotional, behavioral, and relational empowerment components. Journal of Community Psychology, 46(1), 58-78. https:// doi.org/10.1002/jcop.21916

Santos, J. V., Gonçalves, G. y Orgambídez-Ramos, A. (2014). Adaptação da escala de Empowerment Psicológico de Spreitzer numa amostra portuguesa. Avaliação Psicológica, 13(3), 325-332.

Schermelleh-Engel, K., Moosbrugger, H. y Muller, H. (2003). Evaluating the fit of structural equation models: tests of significance and descriptive goodness-of-fit measures. Methods of Psychological Research Online, 8(2), 23-74.

Schumaher, M. L. N., Milani, D. y Alexandre, N. M. C. (2019). Psychometric properties evaluation of the Psychological Empowerment Instrument in a Brazilian context. Journal of Nursing Management, 27(2), 404-413. https:// doi.org/10.1111/jonm.12701

Seibert, S. E., Wang, G. y Courtright, S. H. (2011). Antecedents and consequences of psychological and team empowerment in organizations: A meta-analytic review. Journal of Applied
Psychology, 96(5), 981-1003. https://doi. org/10.1037/a0022676

Spreitzer, G. M. (1995). Psychological Empowerment in the Workplace: Dimensions, Measurement, and Validation. Academy of Management Journal, 38, 1442-1465 https://doi. org/10.2307/256865

Spreitzer, G. M. (1996). Social structural characteristics of psychological empowerment. Academy of Management Journal, 39, 483-504. https://doi.org/10.2307/256789

Sun, N., Li, Q. J., Lv, D. M., ..., y An, X. M. (2011). The psychometric properties of the Chinese version of the Problem Areas in Psychological empowerment Scale (PES): scale development. Journal of Clinical Nursing, 20(3-4), 369-376 https://doi.org/10.1111/j.13652702.2010.03519.x

Thomas, K. W. y Velthouse, B. A. (1990). Cognitive Elements of Empowerment: An "Interpretive" Model of Intrinsic Task Motivation. Academy of Management Review, 15, 666-681. https://doi.org/10.5465/amr.1990.4310926

Uner, S. y Turan, S. (2010). The construct validity and reliability of the Turkish version of Spreitzer's psychological empowerment scale. BMC Public Health, 10, 117. https://doi. org/10.1186/1471-2458-10-117.

Zhang, X., Ye, H. y Li, Y. (2018). Correlates of structural empowerment, psychological empowerment and emotional exhaustion among registered nurses: A meta-analysis. Applied Nursing Research, 42, 9-16. https:// doi.org/10.1016/j.apnr.2018.04.006

Recibido: 4 de junio de 2020 Aceptado: 28 de septiembre de 2021 
Anexo

\section{Versión española del Psychological Empowerment Instrument (Spreitzer, 1995)}

A continuación, indique el grado en el que cada una de las siguientes condiciones de trabajo se da en su puesto de trabajo. Para ello, escriba el número correspondiente al final de cada frase teniendo en cuenta la siguiente escala.

\begin{tabular}{|c|c|c|c|c|c|c|}
\hline 1 & 2 & 3 & 4 & 5 & 6 & 7 \\
\hline Muy poco & Poco & Regular & Suficiente & Bastante & Mucho & Demasiado \\
\hline
\end{tabular}

1. Tengo confianza en mi capacidad para hacer mi trabajo

2. El trabajo que hago es importante para mí

3. Tengo bastante autonomía para decidir cómo hacer mi trabajo

4. Mi influencia sobre lo que ocurre en mi departamento o área es alta

5. Las actividades laborales tienen un significado personal para mí

6. Tengo mucho control sobre lo que sucede en mi departamento

7. Puedo decidir por mí mismo/a cómo voy a realizar mi propio trabajo

8. Tengo bastantes oportunidades de independencia y libertad en la forma de hacer mi trabajo

9. He adquirido las habilidades necesarias para mi trabajo

10. El trabajo que hago tiene sentido para mí

11. Tengo bastante influencia sobre lo que ocurre en mi departamento

12. Estoy seguro de mí mismo/a acerca de mis capacidades para realizar las actividades de mi trabajo

13. Tengo la oportunidad de utilizar la iniciativa personal para llevar a cabo mi trabajo 